\title{
Maneuvering in Near-Earth Space with the Use of the Collinear Libration Points
}

\author{
Alexander Shmyrov and Dzmitry Shymanchuk \\ Applied Mathematics and Control Processes \\ Saint-Petersburg State University \\ Universitetskii prospect 35, Petergof, Saint-Petersburg, Russia, 198504 \\ Email: ashmyrov@yandex.ru, shymanchuk@mail.ru
}

\begin{abstract}
The use of the neighborhood of collinear libration point $\left(L_{1}\right.$ or $\left.L_{2}\right)$ of the Sun-Earth system has long been of practical importance in connection with projects implemented by NASA and ESA. A celestial body motion is considered in a rotating frame within the Hill's problem of the circular restricted three-body problem. It is known that collinear libration points are unstable but its instability can be used as a positive factor for maneuvering a celestial body in the near-Earth space. For example, it may be used to solve the problem of comet and asteroid hazard to monitor space objects posing threat to the Earth. We offer a methodology for constructing control algorithms for the orbital motion of a celestial body. This methodology is based on the properties of a specially introduced phase variables function called "hazard function". Numerical simulation results are given.
\end{abstract}

\section{INTRODUCTION}

Comprehension of the real collision threat to Earth with a large celestial body can be attributed to the middle 90's of the XXth century when astronomers could directly observe falling fragments of Comet Shoemaker-Levy 9 on Jupiter. Since that time, astronomical observations, as well as discoveries of new objects in the Solar System, which may pose a threat to the Earth had intensified. One of solutions to the problem of comet and asteroid hazard is impact on the menacing object. In [1] a suitable asteroid of a sufficiently small size with controlled orbital motion is proposed to be used. Such controlled asteroid could significantly affect the trajectory of the dangerous asteroid and prevent its impact to the Earth. To reduce the energy consumption, it is supposed to use gravitational maneuvering. Here, the instability property of the collision trajectory is used. In our paper we propose using a different type of instability, in particular, the instability of collinear libration points of the Sun-Earth system [2]. This type of instability may be used for interception maneuvering.

To describe the motion of a celestial body, a modified mathematical model of the circular restricted three-body problem is used [3].

Investigating the orbital motion in the neighborhood of the collinear libration point of the Sun-Earth system has intensified with respect to projects (ISEE-3, SOHO, WIND, ACE, Genesis, etc.) implemented by the National Aeronautics and Space Administration (NASA) and the European Space Agency (ESA).

Using the theoretical properties of collinear libration points caused many projects connected with space exploration and protection of the Earth from potentially dangerous space objects. Since the libration points $L_{1}$ and $L_{2}$ are unstable, the problem of retention or celestial body stabilization in the neighborhood of these points [3]-[7] occurs. In some cases instability of collinear libration point can be a positive factor for space maneuvering with relatively small energy consumption [8]-[10]. These maneuvers may occur during sufficiently large period of time caused by gravitational forces affect and other disturbing factors.

It is well known that a particular solution to the restricted three bodies problem is the motion in the ecliptic plane [11]. In our study the properties of planar motion provide instability of $L_{1}$. On the other hand the orbits of dangerous celestial bodies can be inclined towards the ecliptic plane. To intercept such celestial bodies, it is necessary the spatial coordinate to vary in a sufficiently large range.

In the paper this property is provided by a specific way of selecting a parking orbit.

\section{Maneuvering Scheme And Problem FORMULATION}

In the proposed maneuvering scheme countering comet and asteroid hazard involves:

1) selection and calculation of the parking orbit;

2) constructing the boost-phase trajectory;

3) description of the interception trajectory.

In this study the parking orbit is chosen in such a way that only a spatial coordinate is changed. Changing plane variables should be small relative to the amplitude of the spatial variables. The boost-phase trajectory is modeled as an instantaneous change of velocity. The impulses are chosen in such a way that an increment of the "hazard function" [4], [5], [7][10] describing the speed of departure from the neighborhood of $L_{1}$ is extremal. These impulses are necessary for modeling a maneuver for departing from the neighborhood of the collinear libration point. Then a numerical integrating equations of uncontrolled orbital motion is provided.

In this paper the main problem is to study motions trajectories after maneuvering with the use of the proposed scheme using numerical modeling and especially the study of the spatial variable behavior. 


\section{EQUATIONS OF MOTION}

Equations of celestial body motion in a rotating frame while using Hill's problem for solar potential can be represented in the following form

$$
\left\{\begin{array}{l}
\dot{x}_{1}=x_{2}+y_{1} \\
\dot{x}_{2}=-x_{1}+y_{2} \\
\dot{x}_{3}=y_{3} \\
\dot{y}_{1}=-\frac{3 x_{1}}{\|\mathbf{x}\|^{3}}+2 x_{1}+y_{2} \\
\dot{y}_{2}=-\frac{3 x_{2}}{\|\mathbf{x}\|^{3}}-x_{2}-y_{1} \\
\dot{y}_{3}=-\frac{3 x_{3}}{\|\mathbf{x}\|^{3}}-x_{3}
\end{array}\right.
$$

where $\mathbf{x}=\left(x_{1} ; x_{2} ; x_{3}\right)$ is coordinate vector of the celestial body and $\mathbf{y}=\left(y_{1} ; y_{2} ; y_{3}\right)$ is an impulse vector [3]. The center of mass of the Earth coincides with the origin of the coordinate system, while the $O x_{1}$ axis is directed along the axis connecting the centers of mass of the Earth and the Sun. $\|\cdot\|$ is the Euclidean norm of a vector. In the considered model the units of time and distance were chosen in such a way that the unit of distance is approximately equal to $1.5 \times 10^{6} \mathrm{~km}$ and the unit of time is 58.0916 days (the year divided by $2 \pi$ ). The libration points $L_{1}$ and $L_{2}$ in the rotating coordinate system are stationary and have coordinates $\mathbf{x}^{*}=(1 ; 0 ; 0), \mathbf{y}^{*}=(0 ; 1 ; 0)$ and $\mathbf{x}^{* *}=(-1 ; 0 ; 0), \mathbf{y}^{* *}=(0 ;-1 ; 0)$, respectively.

Unit of velocity in the considered model is $298.057 \mathrm{~m} / \mathrm{s}$ $\approx 9.94211 \times 10^{-7}$ of light velocity in vacuum.

System (1) is Hamiltonian where the Hamiltonian $H$

$$
H=\frac{1}{2}\|\mathbf{y}\|^{2}-\frac{3}{\|\mathbf{x}\|}-\frac{3}{2} x_{1}^{2}+\frac{\|\mathbf{x}\|^{2}}{2}+x_{2} y_{1}-x_{1} y_{2} .
$$

To select a parking orbit and initial impulse the properties of the linear approximation of equation system (1) in the neighborhood of $L_{1}$ are necessary.

Linearized motion equations in the neighborhood of $L_{1}$ can be represented in the following form

$$
\left\{\begin{array}{l}
\dot{x}_{1}=x_{2}+y_{1} \\
\dot{x}_{2}=-x_{1}+y_{2} \\
\dot{x}_{3}=y_{3} \\
\dot{y}_{1}=8\left(x_{1}-1\right)+\left(y_{2}-1\right) \\
\dot{y}_{2}=-4 x_{2}-y_{1} \\
\dot{y}_{3}=-4 x_{3}
\end{array}\right.
$$

The matrix of linearized system (2) has the following eigenvalues:

$$
\begin{aligned}
& \lambda_{1,2}= \pm \sqrt{1+2 \sqrt{7}}= \pm l \\
& \lambda_{3,4}= \pm i \sqrt{2 \sqrt{7}-1}= \pm i \omega_{\mathbf{e}} \\
& \lambda_{5,6}= \pm 2 i= \pm i \omega_{\mathbf{n}}
\end{aligned}
$$

Positivity of the eigenvalue $\lambda_{1}$ implies instability of the libration point $L_{1}$.
Further the solution of linearized system (2) can be represented in the following form

$$
\left\{\begin{array}{l}
x_{1}-1=b_{1}^{1}\left(c_{1} e^{l t}+c_{2} e^{-l t}\right)+\mathcal{A}_{\mathbf{e}} \sin \left(\omega_{\mathbf{e}} t+\varphi_{\mathbf{e}}\right), \\
x_{2}=b_{1}^{2}\left(c_{1} e^{l t}-c_{2} e^{-l t}\right)-\kappa_{1} \mathcal{A}_{\mathbf{e}} \cos \left(\omega_{\mathbf{e}} t+\varphi_{\mathbf{e}}\right), \\
x_{3}=\mathcal{A}_{\mathbf{n}} \sin \left(\omega_{\mathbf{n}} t+\varphi_{\mathbf{n}}\right), \\
y_{1}=b_{1}^{3}\left(c_{1} e^{l t}-c_{2} e^{-l t}\right)+\kappa_{2} \mathcal{A}_{\mathbf{e}} \cos \left(\omega_{\mathbf{e}} t+\varphi_{\mathbf{e}}\right), \\
y_{2}-1=b_{1}^{4}\left(c_{1} e^{l t}+c_{2} e^{-l t}\right)+\kappa_{3} \mathcal{A}_{\mathbf{e}} \sin \left(\omega_{\mathbf{e}} t+\varphi_{\mathbf{e}}\right), \\
y_{3}=\kappa_{4} \mathcal{A}_{\mathbf{n}} \cos \left(\omega_{\mathbf{n}} t+\varphi_{\mathbf{n}}\right),
\end{array}\right.
$$

where $c_{1}, c_{2}, \mathcal{A}_{\mathbf{e}}, \varphi_{\mathbf{e}}, \mathcal{A}_{\mathbf{n}}, \varphi_{\mathbf{n}}$ can be defined by initial conditions; $l, \omega_{\mathbf{e}}, \omega_{\mathbf{n}}, b_{1}^{1}, b_{1}^{2}, b_{1}^{3}, b_{1}^{4}, \kappa_{1}, \kappa_{2}, \kappa_{3}, \kappa_{4}$ are model parameters.

Eigenvector matrix of system (2) corresponding to the eigenvalue $\lambda_{1}$ has the form

$$
\mathbf{b}_{1}=\left(b_{1}^{1} ; b_{1}^{2} ; 0 ; b_{1}^{3} ; b_{1}^{4} ; 0\right)=\left(\lambda_{1}^{2}+5 ; \frac{\lambda_{1}^{2}-3}{\lambda_{1}} ; 0 ; \frac{\lambda_{1}^{2}+3}{\lambda_{1}} ; 2 ; 0\right) \text {. }
$$

Let us denote

$$
d_{1}=\mathbf{b}_{1} \mathbf{z}
$$

where $\mathbf{z}=\left(x_{1}-1 ; x_{2} ; x_{3} ; y_{1} ; y_{2}-1 ; y_{3}\right)$ is a vector-column.

Equations (2) have an invariant 5-dimensional manifold filled with bounded trajectories approaching asymptotically to the two-frequency oscillations with frequencies $\omega_{\mathbf{e}}$ and $\omega_{\mathbf{n}}$. This manifold is defined by equation

$$
d_{1}=0,
$$

where function $d_{1}$ is called "hazard function".

\section{CONSTRUCTING A PARKING ORBIT}

In the middle 60's Farquhar proposed using orbits in the neighborhood of libration point $L_{1}$ as an ideal location for continuous monitoring the interplanetary environment. For example, Farquhar constructed a trajectory of the first libration point mission (ISEE-3) [12]. In this project it was proposed using the orbit at $\mathcal{A}_{\mathbf{n}}=120000 \mathrm{~km}$. In the SOHO project the value $\mathcal{A}_{\mathbf{n}}$ was of the same order. In the projects ACE and WMAP the orbits in the neighborhood of the collinear libration points $L_{1}$ and $L_{2}$ where $\mathcal{A}_{\mathbf{n}}=157000 \mathrm{~km}$ were suggested to be used.

In this paper the parking orbits are constructed in the neighborhood of the collinear libration point $L_{1}$ with values $\mathcal{A}_{\mathbf{n}}=150000 \mathrm{~km}$ and $\mathcal{A}_{\mathbf{e}}=0 \mathrm{~km}$. Within considered mathematical model the value $\mathcal{A}_{\mathbf{n}}$ is approximately equal to 0.1 distance units, then an approximate analytical representation in the form of power series with respect to a parameter $\varepsilon$ may be used for the parking orbits. Considering expansion terms till the second order of smallness the solution of the system (1) can be written as

$$
\left\{\begin{array}{l}
x_{1}(t, \varepsilon)=1+\varepsilon^{2}\left(-\frac{1}{4}-\frac{13}{116} \cos (4 t+2 \alpha)\right)+o\left(\varepsilon^{2}\right) \\
x_{2}(t, \varepsilon)=\varepsilon^{2} \frac{2}{29} \sin (4 t+2 \alpha)+o\left(\varepsilon^{2}\right) \\
x_{3}(t, \varepsilon)=\varepsilon \cos (2 t+\alpha)+o\left(\varepsilon^{2}\right) \\
y_{1}(t, \varepsilon)=\varepsilon^{2} \frac{11}{29} \sin (4 t+2 \alpha)+o\left(\varepsilon^{2}\right) \\
y_{2}(t, \varepsilon)=1+\varepsilon^{2}\left(-\frac{1}{4}+\frac{19}{116} \cos (4 t+2 \alpha)\right)+o\left(\varepsilon^{2}\right) \\
y_{3}(t, \varepsilon)=-2 \varepsilon \sin (2 t+\alpha)+o\left(\varepsilon^{2}\right)
\end{array}\right.
$$

Within the accuracy of up to the second order of smallness of $\varepsilon$ plane variables have oscillation frequency 4 , while spatial 


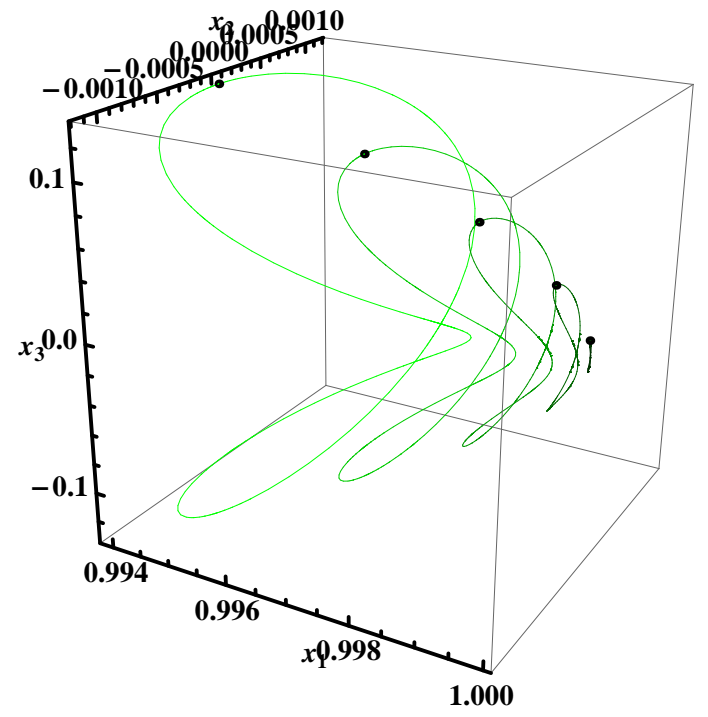

Fig. 1. $L_{1}$ parking orbits around the Sun-Earth system with $\varepsilon_{i}=0.01+$ $0.03 i, i=0, \ldots, 4$

variables have 2. The solutions obtained in [13] are called Lyapunov vertical trajectories.

\section{Selecting the Initial Impulse}

Let a celestial body be moving along the parking orbit described approximately by equations (3). In view of linearized system of motion equations (2) the behavior of "hazard function" $d_{1}$ on the trajectories can be represented as

$$
d_{1}(t)=d_{1}\left(t_{0}\right) e^{\lambda_{1}\left(t-t_{0}\right)},
$$

where $t_{0}$ is initial time.

On the parking orbit the value of the "hazard function" is very small. To provide speedy departure from the neighborhood of libration point $L_{1}$, initial value of $d_{1}$ should be changed. It can be made with the use of small impact changing the value of the impulses $y_{1}$ and $y_{2}$ instantly.

Let the vector of initial impulse change $\left(\Delta y_{1} ; \Delta y_{2}\right)$ be subject to restriction $\Delta y_{1}^{2}+\Delta y_{2}^{2}=\Delta y^{2}$. Then the vector $\left(\Delta y_{1} ; \Delta y_{2}\right)$ should be parallel to the vector $\left(b_{1}^{3} ; b_{1}^{4}\right)$ in order to obtain the highest increment of the "hazard function". From here we get

$$
\left\{\begin{array}{l}
\Delta y_{1}=\Delta y \frac{ \pm b_{1}^{3}}{\sqrt{\left(b_{1}^{3}\right)^{2}+\left(b_{1}^{4}\right)^{2}}} \\
\Delta y_{2}=\Delta y \frac{ \pm b_{1}^{4}}{\sqrt{\left(b_{1}^{3}\right)^{2}+\left(b_{1}^{4}\right)^{2}}} .
\end{array}\right.
$$

Some examples are given further.

\section{NUMERICAL RESUlTS}

Let us present the results of numerical modeling of celestial body motion after departing the neighborhood of $L_{1}$ at different variations of initial impact. The results of numerical experiments are illustrated in the Figures below.

In Fig. 2 and Fig. 3 it is shown that small impact initiates the celestial body to perform an extended maneuver in the

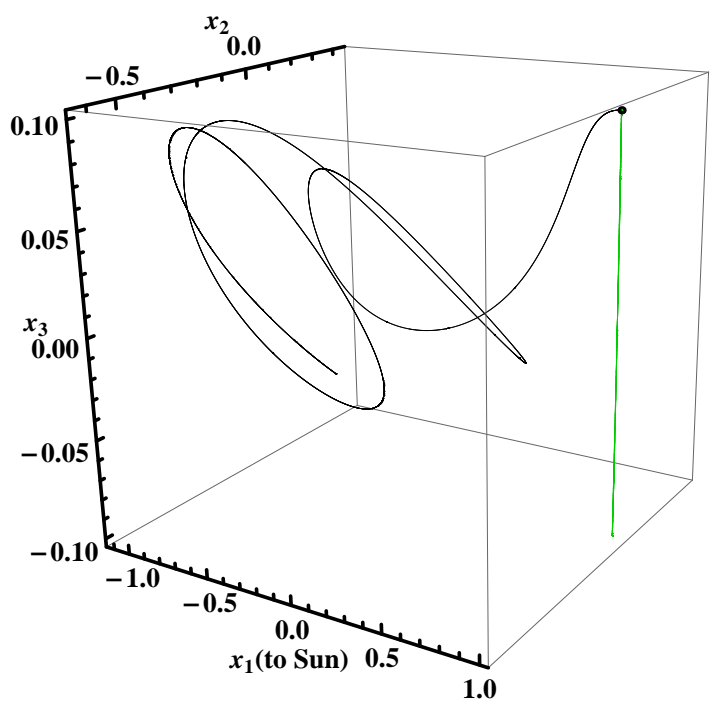

Fig. 2. The flight trajectory of the celestial body from the neighborhood of the parking orbit $L_{1}(\varepsilon=0.1)$ in the neighborhood of libration point $L_{2}$ (initial impulse $\Delta y_{1}=-0.803$ and flight time $T=7$ )

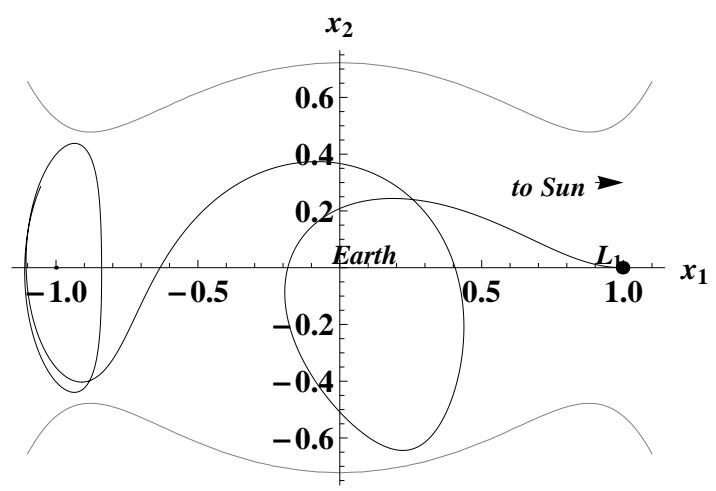

Fig. 3. Projection of celestial body orbit (shown in Fig. 2) on an ecliptic plane

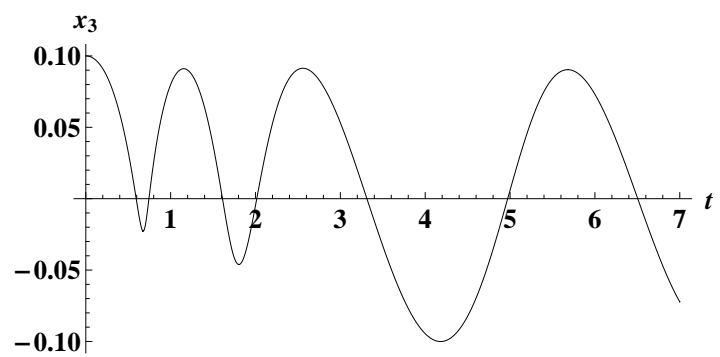

Fig. 4. Graph of dependence of $x_{3}$ from time in the trajectory of Fig. 2.

near-Earth space; the time of motion along trajectories is 1.17 years. In Fig. 5 and Fig. 6 the celestial body maneuvers with returning back to the neighborhood of libration point $L_{1}$, which corresponds to a longer period of time - about 1.67 years. Fig. 4 and Fig. 7 show the graph of spatial coordinate $x_{3}$ and time of flight maneuvers, which are shown in Fig. 2 and Fig. 5, respectively. The initial time and method of applying the control impact are selected in accordance with the maneuver 


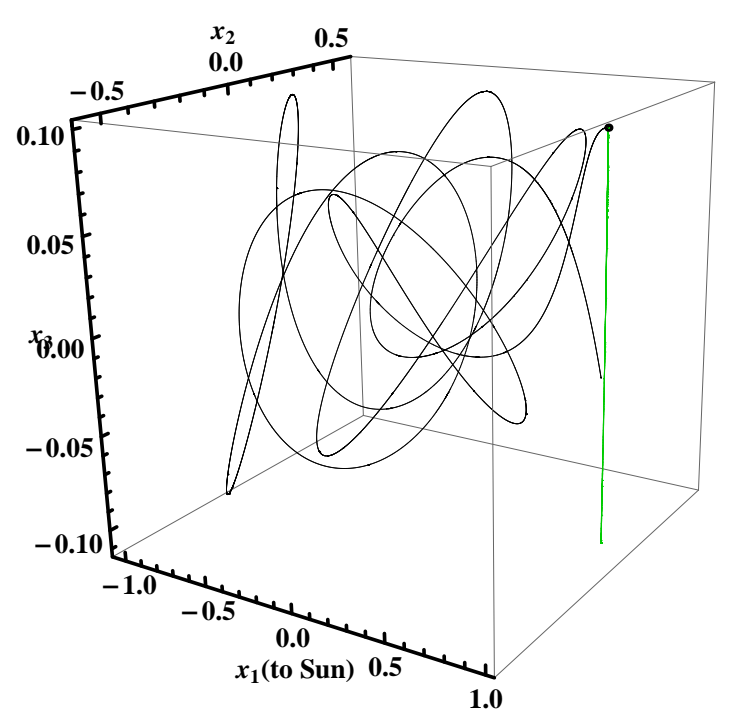

Fig. 5. Celestial body flight trajectory from the neighborhood of the parking orbit $L_{1}(\varepsilon=0.1)$ in the neighborhood of libration point $L_{1}$ (initial impulse $\Delta y_{1}=-0.421$ and flight time $T=10$ )

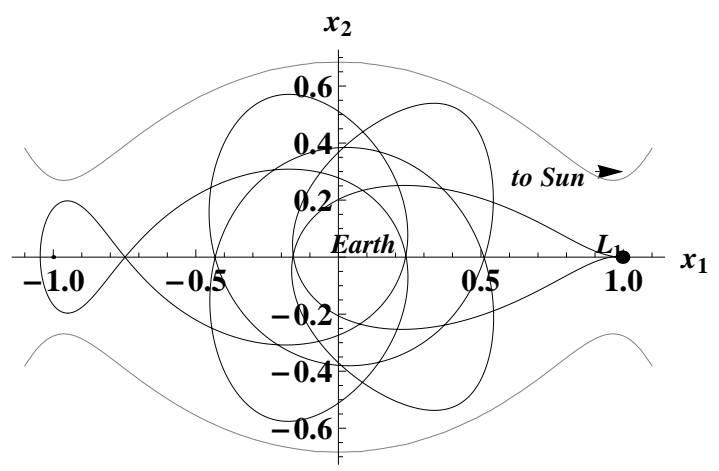

Fig. 6. Projection of celestial body orbit (shown in Fig. 5) on an ecliptic plane

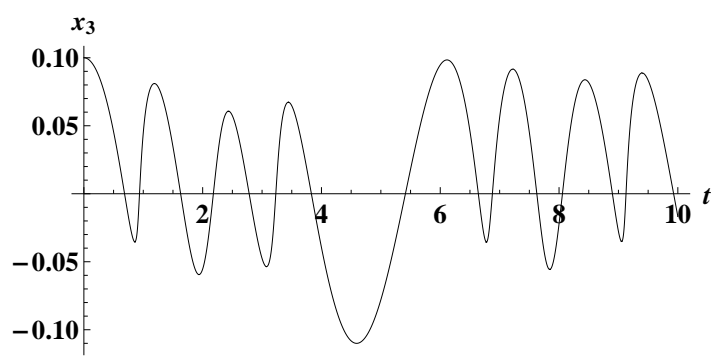

Fig. 7. Graph of dependence of $x_{3}$ from time in the trajectory of Fig. 5 .

scheme provided.

Fig. 8 shows how the celestial body maneuvers using a small initial impulse $\Delta y=0.298 \mathrm{~m} / \mathrm{s}$ in order to sufficiently quickly depart the neighborhood of the parking orbit of the collinear libration point $L_{1}$. For example, to bring the celestial body from low-Earth orbit to a parabolic one it is necessary to give impulse approximately equal to $3.3 \mathrm{~km} / \mathrm{s}$, which is the difference between the second and the first cosmic velocities.

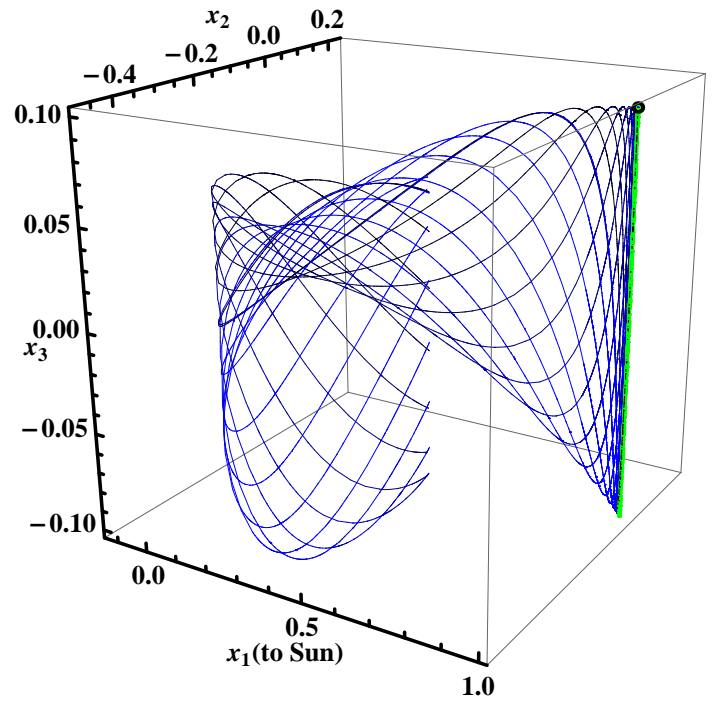

Fig. 8. The trajectories of speedy departure from the neighborhood of the parking orbit of collinear libration point $L_{1}(\varepsilon=0.1)$ to the near-Earth space. Initial impulse $\Delta y_{1}=-0.879939 \times 10^{-3}, \Delta y_{2}=-0.475087 \times 10^{-3}$ and flight time $T=4$

Motion along the trajectory corresponds to a time interval of about 8 months. Fig. 8 shows the behavior of the mentioned above trajectories depending on the celestial body position in the parking orbit of collinear libration point $L_{1}$.

\section{CONCLUSION}

This study on the orbital motion of a celestial body shows that a small initial impulse can provide sufficiently rapid departure from the neighborhood of the collinear libration point $L_{1}$. Given examples of orbital motion show the theoretical possibility of celestial body maneuvering in the near-Earth space using the neighborhoods of collinear libration points $L_{1}$ and $L_{2}$. In these cases instability is achieved by the use of sufficiently small control impacts, so it allows to change the trajectory of the celestial body significantly with minimal energy costs. Theoretically the energy costs for removal of the controlled object from an unstable state can be arbitrarily small. It makes attractive our maneuvering scheme in order to control the orbital motion of celestial bodies with a large mass. In particular, these bodies may be used to impact on hazardous asteroids. From given results it is clear that the instability of a collinear libration point has a significant impact on the behavior of plane variables $x_{1}, x_{2}, y_{1}, y_{2}$ and has virtually no effect on the changes of spatial variables $x_{3}, y_{3}$. There are oscillations with amplitude close to the amplitude $\mathcal{A}_{\mathbf{n}}$ of the parking orbit (see. Fig. 2 and Fig. 4, Fig. 5 and Fig. 7) are characteristic of $x_{3}$.

\section{ACKNOWLEDGMENT}

The authors acknowledge Saint-Petersburg State University for a research grant 9.37.345.2015

\section{REFERENCES}

[1] N. A. Eismont et al., "On the possibility of the guidance of small asteroids to dangerous celestial bodies using the gravity-assist maneuver," Solar System Research, vol. 47, no. 4, pp. 325-333. 2013. 
[2] A.P. Markeev, "Libration points in celestial mechanics and cosmodynamics," Moscow, Nauka. (in Russian) 1978.

[3] V. A. Shmyrov, "Stabilization of controlled space vehicle orbital motion in the neighborhood of collinear libration point L1," Bulletin of St. Petersburg University. Series 10, no. 2, pp. 193-199, (in Russian) 2005.

[4] A. S. Shmyrov, V. A. Shmyrov, "Synthesis of an optimal control of orbital movement in a neighborhood of a collinear libration point," Bulletin of St.-Petersburg University. Series 1, no. 4, pp. 139-146, (in Russian) 2012.

[5] A. Shmyrov, V. Shmyrov, "Controllable orbital motion in a neighborhood of collinear libration point," Applied Mathematical Sciences, vol. 8 (9-12), pp. 487-492, 2014.

[6] A. Shmyrov, V. Shmyrov, "Method of Lyapunov functions for controllable Hamiltonian systems," 2014 20th International Workshop on Beam Dynamics and Optimization, BDO 2014, art. no. 6890078, 2014.

[7] D. V. Shymanchuk, "Modelling of orbital controlled motion of a spacecraft in the neighborhood of the collinear libration point $L_{1}$," Bulletin of St.-Petersburg University. Series 10, no. 3, pp. 86-92, (in Russian) 2010
[8] D. V. Shymanchuk, A.S. Shmyrov, "Construction of trajectory of the return in the neighborhood of the collinear libration point of the SunEarth system," Bulletin of St.-Petersburg University. Series 10, no. 2, pp. 76-85, (in Russian) 2013.

[9] F. M. Kulakov, A. S. Shmyrov, D. V. Shymanchuk, "Supervisory remote control of space robot in an unstable libration point," Proceedings of the 2013 IEEE 7th International Conference on Intelligent Data Acquisition and Advanced Computing Systems, IDAACS, vol. 2, pp. 925-928, 2013.

[10] A. Shmyrov, V. Shmyrov, D. Shymanchuk, "Prospects for the use of space robots in the neighborhood of the libration points," Applied Mathematical Sciences, (49-52), pp. 2465-2471, 2014.

[11] A. B. Batkhin, "Generating planar periodic orbits Hill problem," Keldysh Institute Preprints, no. 47, (in Russian) 2010.

[12] R. W. Farquhar, D. P. Muhonen, D. L. Richardson, "Mission Design for a Halo Orbiter of the Earth," J. of Spacecraft and Rockets, vol. 14, no. 3, pp. 170-177, 1977.

[13] M. Ceriorry, J. P. S. Cuartiellez, "Orbit control of asteroids in libration point orbits for resource exploitation," Proceedings of 64th International Astronautical Congress, Beijing, China. Paper IAC-13-C1.4.3, 2013. 